\title{
FIXED POINTS OF A CERTAIN CLASS OF MAPPINGS IN SPACES WITH UNIFORMLY NORMAL STRUCTURE
}

\author{
JONG SOO JUNG \\ Department of Mathematics, Dong-A University \\ Pusan 607-714, KOREA \\ E-mail address: jungjs@seunghak.donga.ac.kr
}

BALWANT SINGH THAKUR

Govt. B. H. S. S. Gariaband

Dist. Raipur, M. P. 493889, INDIA

and

DAYA RAM SAHU

Govt. H. S. S. Kumhari

Dist. Durg, M. P. 490042, INDIA

(Received September 12, 1996 and in revised form May 4, 1997)

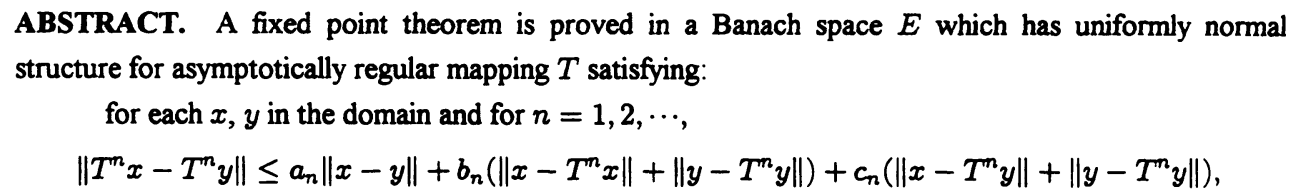
where $a_{n}, b_{n}, c_{n}$ are nonnegative constants satisfying certain conditions. This result generalizes a fixed point theorem of Górnicki [1].

KEY WORDS AND PHRASES: Uniformly normal structure, asymptotic regularity, fixed point. 1991 AMS SUBJECT CLASSIFICATION CODES: 47H10.

\section{INTRODUCTION}

Let $E$ be a Banach space and $K$ a nonempty, bounded, closed and convex subset of $E$. A mapping $T: K \rightarrow K$ is said to be nonexpansive if $\|T x-T y\| \leq\|x-y\|$ for all $x, y \in K$. Browder [2], Göhde [3] and Kirk [4] proved independently that if $E$ is uniformly convex, then $T$ always has a fixed point in $K$ (see also Goebel [5]). Now, it is important (cf. [4]) that if one assumes $T$ to be Lipschitzian with Lipschitz constant $k>1$, then $T$ need not have a fixed point, even if $E$ is a Hilbert space and $k$ is an arbitrary near 1. However, there are classes of transformations which lie between the nonexpansive transformation and those with Lipschitz constant $k>1$ for which fixed point theorems do exist; in particular, the asymptotically nonexpansive mappings (cf. [6]) form such a class. These are mappings $T: K \rightarrow K$ having the property that $T^{n}$ has Lipschitz constant $k_{n}$ with $k_{n} \rightarrow 1$ as $n \rightarrow \infty$.

In this paper, we obtain a fixed point theorem for the class of mappings whose nth iterate $T^{n}$ satisfy:

$$
\left\|T^{n} x-T^{n} y\right\| \leq a_{n}\|x-y\|+b_{n}\left(\left\|x-T^{n} x\right\|+\left\|y-T^{n} y\right\|\right)+c_{n}\left(\left\|x-T^{n} y\right\|+\left\|y-T^{n} x\right\|\right)
$$

for each $x, y \in K$ and $n=1,2, \cdots$, where $a_{n}, b_{n}, c_{n}$ are nonnegative constants such that there exists an integer $n_{0}$ such that $b_{n}+c_{n}<1$ for all $n \geq n_{0}$. This class of mappings are more general than nonexpansive mappings. Also by taking $b_{n}=c_{n}=0$ it will be seen that this class of mappings are more general than asymptotically nonexpansive mappings. Our result improves and extends the results of Górnicki [1] and others. 


\section{PRELIMINARIES}

The concept of uniformly normal structure is due to Gillespie and Williams [7]. A Banach space $E$ has uniformly normal structure if

$$
N(E)=\sup \left\{r_{K}(K): K \subset E \text { is convex and } \operatorname{diam} K=1\right\}<1,
$$

where

$$
r_{K}(K)=\inf \{\sup \{\|x-y\|: y \in K\}: x \in K\} .
$$

It was proved in [8], [9] that $N(E) \leq 1-\delta_{E}(1)$; thus $\epsilon_{0}(E)<1$ implies uniformly normal structure, where $\delta_{E}(\cdot)$ is the modulus of convexity of $E$ and $\epsilon_{0}(E)$ is the characteristic of convexity of $E$. Yu [10] proved that if $E$ is a uniformly smooth space, then $E$ has a uniformly normal structure. Also, in [11] it was proved that uniformly normal structure does not necessarily imply that the space has good geometric properties.

The following lemma is needed to prove our main result:

LEMMA 1 [12]. Let $K$ be a nonempty closed convex subset of a Banach space $E$ and let $\left\{n_{2}\right\}$ be an increasing sequence of natural numbers. Assume that $T: K \rightarrow K$ is an asymptotically regular mapping such that for some $m \in \mathbb{N}, T^{m}$ is continuous. If

$$
\lim _{i \rightarrow \infty}\left\|z-T^{m_{i}} x\right\|=0
$$

for some $x \in K$ and $z \in K$, then $T z=z$.

\section{MANN RESULTS}

Now we state and prove our main result:

THEOREM 1. Let $K$ be a nonempty closed convex subset of a Banach space $E$ which has uniformly normal structure, i.e. $N(E)<1$. Let $T: K \rightarrow K$ be as asymptotically regular mapping which holds the inequality (1) such that $(\alpha+\beta) \cdot \gamma \cdot N(E)<1$, where

$$
\begin{gathered}
\alpha=\liminf _{n \rightarrow \infty} \frac{a_{n}+c_{n}}{1-c_{n}} \\
\beta=\liminf _{n \rightarrow \infty} \frac{b_{n}}{1-c_{n}}
\end{gathered}
$$

and

$$
\gamma=\liminf _{n \rightarrow \infty} \frac{a_{n}+c_{n}}{1-c_{n}-b_{n}} .
$$

Suppose that there is a $z_{0}$ in $K$ for which $\left\{T^{n} z_{0}\right\}$ is bounded. Then $T$ has a fixed point in $K$.

PROOF. Let $\left\{n_{i}\right\}$ be a sequence of natural numbers such that

$$
\begin{gathered}
\alpha=\liminf _{n \rightarrow \infty} \frac{a_{n}+c_{n}}{1-c_{n}}=\lim _{i \rightarrow \infty} \frac{a_{n_{1}}+c_{n_{n}}}{1-c_{n_{n}}} \\
\beta=\liminf _{n \rightarrow \infty} \frac{b_{n}}{1-c_{n}}=\lim _{i \rightarrow \infty} \frac{b_{n_{1}}}{1-c_{n_{n}}}
\end{gathered}
$$

and

$$
\gamma=\liminf _{n \rightarrow \infty} \frac{a_{n}+c_{n}}{1-c_{n}-b_{n}}=\lim _{i \rightarrow \infty} \frac{a_{n_{1}}+c_{n_{1}}}{1-c_{n_{1}}-b_{n_{n}}} .
$$

Since $\left\{T^{n} z_{0}\right\}$ is bounded (and hence $\left\{T^{n} z\right\}$ is bounded for any $z$ in $K$ ), by Lemma 1 , we can inductively construct a sequence $\left\{z_{m}\right\}$ such that $z_{m}$ is the unique asymptotic center of the sequence $\left\{T^{m_{1}} z_{m-1}\right\}_{2 \geq 1}$ with respect to the functional

$$
\underset{n \rightarrow \infty}{\limsup }\left\|x-T^{m_{n}} z_{m-1}\right\|
$$


over $x$ in $K$. Now for each $m \geq 1$, we set

$$
D_{m}=\lim _{i \rightarrow \infty}\left\|z_{m}-T^{m_{i}} z_{m}\right\|
$$

and

$$
r_{m}=\lim _{i \rightarrow \infty}\left\|z_{m+1}-T^{m_{i}} z_{m}\right\|
$$

Using (1), we have

$$
\begin{aligned}
& \left\|T^{m_{1}} x-T^{n_{\jmath}} y\right\| \leq\left\|T^{n_{1}} x-T^{n_{1}+n_{\jmath}} y\right\|+\left\|T^{n_{1}+n_{j}} y-T^{n_{\jmath}} y\right\| \\
& \leq a_{n_{1}}\left\|x-T^{n_{3}} y\right\|+b_{n_{1}}\left(\left\|x-T^{n_{1}} x\right\|+\left\|T^{n_{1}} y-T^{n_{1}+n_{j}} y\right\|\right) \\
& +c_{n_{1}}\left(\left\|x-T^{n_{1}+n_{y}} y\right\|+\left\|T^{n_{3}} y-T^{n_{2}} x\right\|\right)+\left\|T^{n_{1}+n_{j}} y-T^{n_{3}} y\right\|
\end{aligned}
$$

implies

$$
\begin{aligned}
\left\|T^{n_{2}} x-T^{n,} y\right\| \leq & \frac{a_{n_{1}}+c_{n_{1}}}{1-c_{n_{1}}} \cdot\left\|x-T^{n_{3}} y\right\|+\frac{b_{n_{1}}}{1-c_{n_{1}}} \cdot\left\|x-T^{n_{1}} x\right\| \\
& +\frac{1+b_{n_{1}}+c_{n_{2}}}{1-c_{n_{1}}} \cdot\left\|T^{n_{3}} y-T^{n_{1}+n_{3}} y\right\| .
\end{aligned}
$$

By inequality (2), the result of Casini and Maluta [13], and the asymptotic regularity of $T$, we have

$$
\begin{aligned}
& r_{m} \leq N(E) \cdot \underset{s \rightarrow \infty}{\limsup }\left(\left\|T^{m_{i}} z_{m}-T^{n,} z_{m}\right\|: n_{i}, n_{j} \geq s\right) \\
& \leq N(E) \cdot \underset{i \rightarrow \infty}{\limsup }\left(\limsup _{j \rightarrow \infty}\left\|T^{m_{i}} z_{m}-T^{n_{j}} z_{m}\right\|\right) \\
& \leq N(E) \cdot \limsup _{z \rightarrow \infty}\left[\operatorname { l i m s u p } _ { j \rightarrow \infty } \left\{\frac{a_{n_{1}}+c_{n_{2}}}{1-c_{n_{1}}} \cdot\left\|z_{m}-T^{m_{\jmath}} z_{m}\right\|+\frac{b_{n_{2}}}{1-c_{n_{1}}} \cdot\left\|z_{m}-T^{m_{1}} z_{m}\right\|\right.\right. \\
& \left.\left.+\frac{1+b_{n_{1}}+c_{n_{1}}}{1-c_{n_{1}}} \cdot \sum_{l=0}^{n_{1}-1} \cdot\left\|T^{n_{1}+l+1} z_{m}-T^{m,+l} z_{m}\right\|\right\}\right]
\end{aligned}
$$

and so

$$
r_{m} \leq(\alpha+\beta) \cdot N(E) \cdot D_{m}, \quad m=0,1, \cdots,
$$

where $N(E)$ is the normal structure coefficient of $E$. Moreover, for $i>1$, we have

$$
\begin{aligned}
& \left\|T^{m_{i}} z_{m}-z_{m}\right\| \leq \limsup _{\jmath \rightarrow \infty}\left\|T^{m_{1}} z_{m}-T^{m_{j}} z_{m-1}\right\| \leq \limsup _{j \rightarrow \infty}\left\{\frac{a_{n_{1}}+c_{m_{1}}}{1-c_{n_{1}}} \cdot\left\|z_{m}-T^{n_{j}} z_{m-1}\right\|\right. \\
& \left.+\frac{b_{m_{1}}}{1-c_{n_{1}}} \cdot\left\|z_{m}-T^{m_{1}} z_{m}\right\|+\frac{1+b_{n_{1}}+c_{n_{1}}}{1-c_{n_{1}}} \cdot \sum_{l=0}^{n_{1}-1}\left\|T^{n_{j}+l+1} z_{m-1}-T^{m_{j}+l} z_{m-1}\right\|\right\} \\
& \leq \frac{a_{n_{1}}+c_{n_{1}}}{1-c_{n_{1}}} \cdot r_{m-1}+\frac{b_{n_{1}}}{1-c_{n_{1}}} \cdot\left\|z_{m}-T^{m_{1}} z_{m}\right\| \\
& \leq \frac{a_{n_{1}}+c_{n_{1}}}{1-b_{n_{1}}-c_{n_{1}}} \cdot r_{m-1}
\end{aligned}
$$

Taking the limit superior as $i \rightarrow \infty$ on each side, by definition of $z_{m}$, we get

$$
\begin{aligned}
D_{m} & \leq \lim _{i \rightarrow \infty}\left(\frac{a_{m_{1}}+c_{m_{1}}}{1-b_{n_{i}}-c_{n_{1}}}\right) \cdot r_{m-1} \\
& \leq \gamma \cdot r_{m-1} .
\end{aligned}
$$

By (3) and (4), we obtain

$$
\begin{aligned}
r_{m} & \leq(\alpha+\beta) \cdot \gamma \cdot N(E) \cdot r_{m-1} \\
& =A \cdot r_{m-1},
\end{aligned}
$$


where $A=(\alpha+\beta) \cdot \gamma \cdot N(E)<1$ by the assumption of the theorem. Since

$$
\left\|z_{m+1}-z_{m}\right\| \leq r_{m}+D_{m} \rightarrow 0
$$

as $m \rightarrow \infty$, it follows that $z_{m}$ is a Cauchy sequence. Let $\lim _{m \rightarrow \infty} z_{m}=z \in K$. Then, we have

$$
\begin{aligned}
& \left\|z-T^{m_{n}} z\right\| \leq\left\|z-z_{m}\right\|+\left\|z_{m}-T^{n_{1}} z_{m}\right\|+\left\|T^{m_{1}} z_{m}-T^{m_{n}} z\right\| \\
& \leq\left\|z-z_{m}\right\|+\left\|z_{m}-T^{m_{n}} z_{m}\right\|+a_{m_{m}}\left\|z_{m}-z\right\| \\
& +b_{n_{i}}\left(\left\|z_{m}-T^{n_{2}} z_{m}\right\|+\left\|z-T^{n_{3}} z\right\|\right)+c_{m_{m}}\left(\left\|z_{m}-T^{n_{i}} z\right\|+\left\|z-T^{m_{i}} z_{m}\right\|\right)
\end{aligned}
$$

and so

$$
\left\|z-T^{m_{1}} z\right\| \leq \frac{1+a_{m_{1}}+2 c_{m_{1}}}{1-b_{n_{1}}-c_{m_{n}}} \cdot\left\|z-z_{m}\right\|+\frac{1+b_{n_{1}}+c_{m_{1}}}{1-b_{n_{1}}-c_{m_{m}}} \cdot\left\|z_{m}-T^{m_{1}} z_{m}\right\|
$$

Taking the limit superior as $i \rightarrow \infty$ on each side, we obtain

$$
\underset{i \rightarrow \infty}{\limsup }\left\|z-T^{n_{2}} z\right\| \leq \limsup _{i \rightarrow \infty} \frac{1+a_{n_{1}}+2 c_{n_{1}}}{1-b_{n_{1}}-c_{n_{1}}} \cdot\left\|z-z_{m}\right\|+\limsup _{i \rightarrow \infty} \frac{1+b_{n_{1}}+c_{m_{1}}}{1-b_{n_{1}}-c_{n_{1}}} \cdot D_{m} \rightarrow 0
$$

as $m \rightarrow \infty$. Therefore we have $T z=z$ by Lemma 1 . This completes the proof.

If we put $b_{n}=c_{n}=0$ in (1), then from Theorem 1, we have the following result.

COROLLARY 1 [1, Theorem 3]. Let $K$ be a nonempty bounded closed convex subset of a Banach space $E$ which has uniformly normal structure, i.e. $N(E)<1$. If $T: K \rightarrow K$ is an asymptotically regular mapping such that

$$
\liminf _{n \rightarrow \infty}\left\|T^{m}\right\|=k<[N(E)]^{-\frac{1}{2}},
$$

then $T$ has a fixed point in $K$.

REMARK. In place of bounded subset of $K$ in [1], we have weaker assumption that there is a $z_{0}$ in $K$ for which $\left\{T^{n} z_{0}\right\}$ is bounded.

\section{REFERENCES}

[1] GÓRNICKI, I., Fixed points of asymptotically regular mappings in spaces with uniformly normal structure, Comment. Math. Univ. Carolinae 32 (4) (1991), 639-643.

[2] BROWDER, F.E., Nonexpansive nonlinear operators in a Banach space, Proc. Nat. Acad. Scl. U.S.A. 54 (1965), 1041-1044.

[3] GÖHDE, O., Zum prinzip der kontraktiven Abbildung, Math. Nachr. 30 (1965), 251-258.

[4] KIRK, W.A., A fixed point theorem for mappings which do not increase distances, Amer. Math. Monthly 72 (1965), 1004-1006.

[5] GOEBEL, K., An elementary proof of the fixed point theorem of Browder and Kirk, Michigan Math. J. 16 (1969), 381-383.

[6] GOEBEL, K. and KIRK, W.A., A fixed point theorem for asymptotically nonexpansive mappings, Proc. Amer. Math. Soc. 35 (1) (1972), 171-174.

[7] GILLESPIE, A.A and WILLIAMS, B.B., Fixed point theorem for nonexpansive mappings on Banach spaces with uniformly normal structure, Appl. Anal. 9 (1979), 121-124.

[8] DANÉS, J., On densifying and related mappings and their applications in nonlinear functional analysis, Theory of Nonlinear Operators Proc. Summer School, Oct. 1972, GDR, Akademie Verlag, 1974, pp. 15-56.

[9] BYNUM, W.L., Normal structure coefficient for Banach space, Pacific J. Math. 86 (1980), 427. 436.

[10] YU, X.T., On uniformly normal structure, Kexue Tongbao 33 (1988), 700-702.

[11] YU, X.T., A geometrically aberrant Banach space with uniformly normal structure, Bull. Austral. Math. Soc. 38 (1988), 99-103.

[12] KRUPPEL, M., Ein Fixpunktentz für asymptotisch reguläre operatoren in gleichmäpig Konvexen Banach-Raumen, Wiss. Z. Padafog. Hochsch. "Liselotte Herrmann" Gustoow, Math-Naturwiss. Fak 25 (1987), 241-246.

[13] CASINI, E. and MALUTA, E., Fixed points of uniformly Lipschitzian mappings in spaces with uniformly normal structure, Nonlinear Anal. TMA 9 (1985), 103-108. 


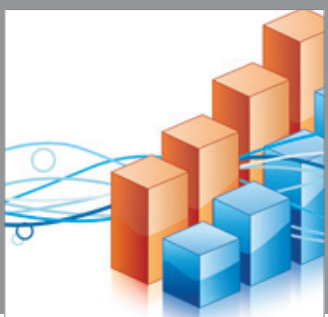

Advances in

Operations Research

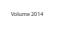

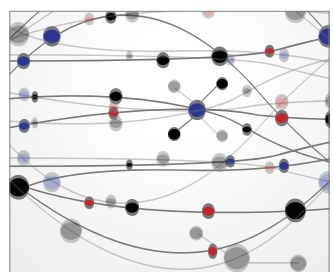

\section{The Scientific} World Journal
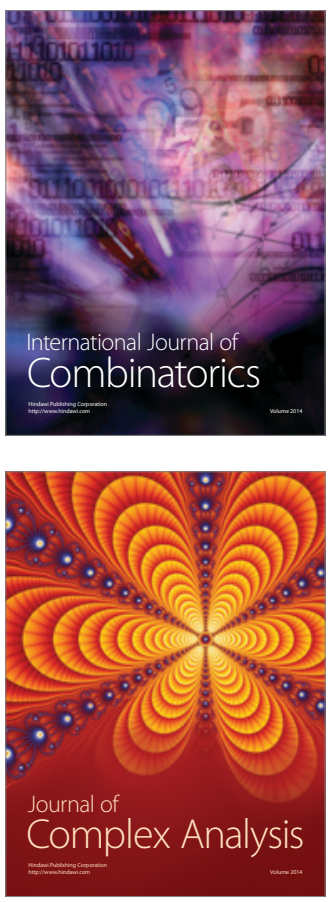

International Journal of

Mathematics and

Mathematical

Sciences
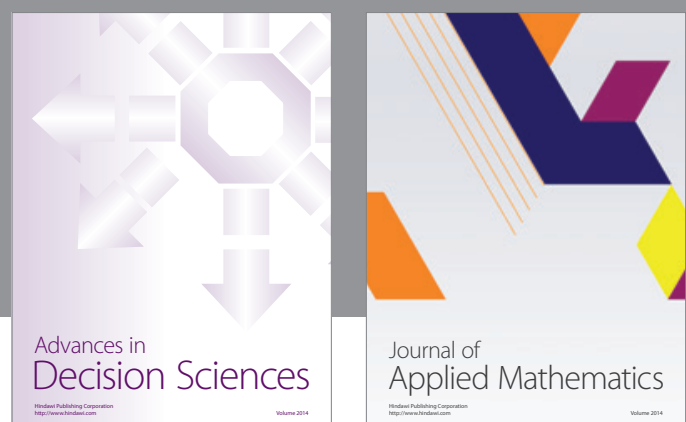

Journal of

Applied Mathematics
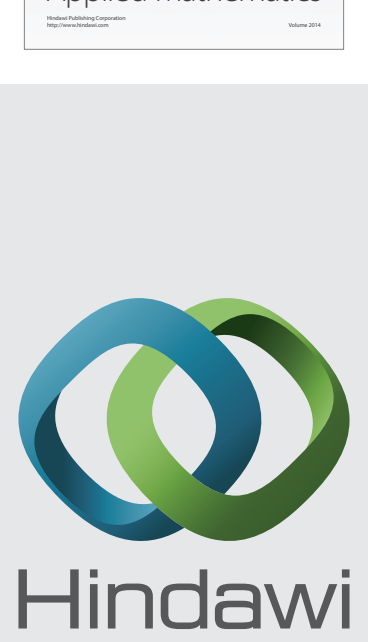

Submit your manuscripts at http://www.hindawi.com
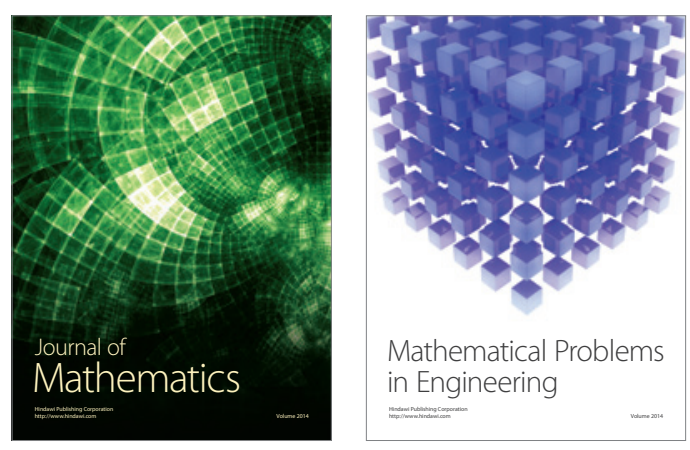

Mathematical Problems in Engineering
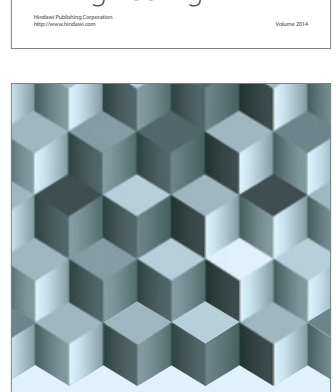

Journal of

Function Spaces
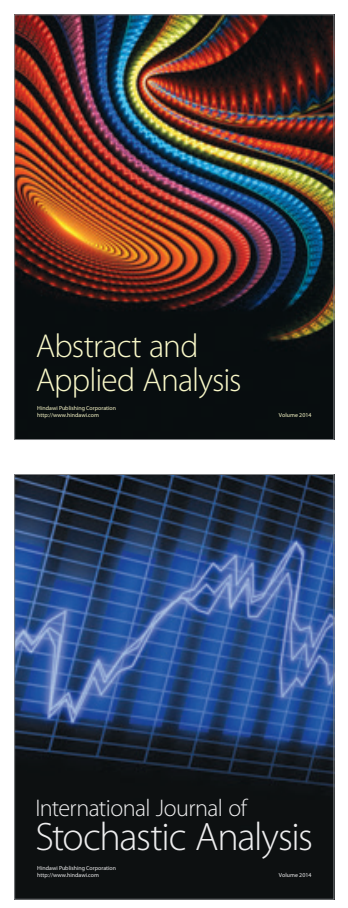

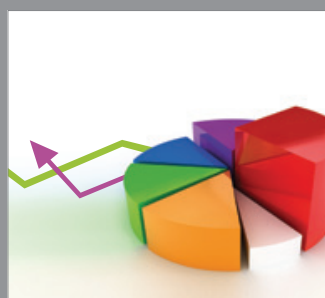

ournal of

Probability and Statistics

Promensencen
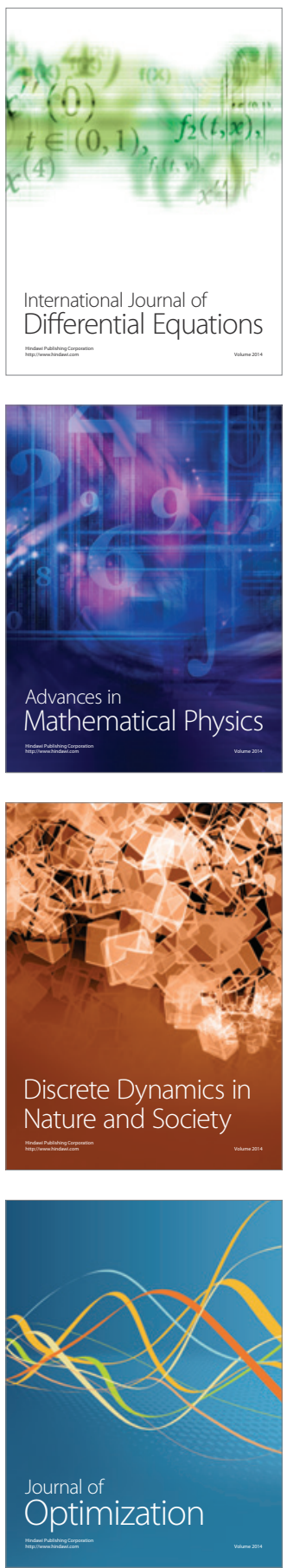